\title{
Measurement of Unwanted Thought Suppression Strategies with the Thought Control Questionnaire in the General Polish Population: A Confirmatory Factor Analysis and Validation
}

This article was published in the following Dove Press journal:

Psychology Research and Behavior Management

\author{
Remigiusz Szczepanowski (D) ${ }^{1,2}$ \\ Ewelina Cichoń ${ }^{2,3}$ \\ Tomasz Niemiec ${ }^{2,3}$ \\ Beata E Andrzejewska (iD ${ }^{2,3}$ \\ Monika Wójta-Kempa (iD)
}

'Department of Public Health, Faculty of Health Sciences, Wroclaw Medical

University, Wroclaw, Poland;

${ }^{2}$ Department of Psychology, Faculty of Applied Studies, Research Unit for Clinical Psychology, University of Lower Silesia, Wroclaw, Poland; ${ }^{3}$ Faculty of Psychology, WSB University in Torun, Torun, Poland
Introduction: Although psychological studies have suggested both the desired and paradoxical effects of unwanted thought suppression, we still know little about this mechanism. It has been proposed that individual differences in using specific strategies to suppress intrusions explain why contradictory effects of suppression are observed. The main aims of the study were to investigate the factor structure of the Polish version of Thought Control Questionnaire (TCQ) and verify whether this structure corresponds to the original version of the TCQ measurement.

Methods: Using the TCQ, which is a 30-item self-report measure, this research investigated individual thought control strategies to suppress intrusive thoughts in the general population. We used parallel analysis and theoretical interpretability to investigate the most appropriate factor structure of the inventory. To examine the validity of the Polish version of TCQ the correlational analysis of TCQ factors with other psychometric scales: Beck Depression Inventory, The State-Trait Anxiety Inventory and The Metacognitions Questionnaire. The internal consistency of the TCQ subscales was also assessed by calculating the Cronbach's alpha reliability coefficients for each factor.

Results: The resulting five-factor solution explained $51.86 \%$ of the total variance. The Polish version of TCQ consisted of five subscales yielding satisfactory reliability values: 1 ) Punishment ( $\alpha=0.725)$; 2) Distraction $(\alpha=0.688)$; 3) Social Control $(\alpha=0.780)$; 4) Worry $(\alpha=0.788 ; 5)$ Re-appraisal $(\alpha=0.70)$.

Conclusion: The five-factor solution was convergent with the dimensions that appeared in the original TCQ version and were observed in the most TCQ adaptations in various countries. Our findings support the psychological construct of thought-control strategies measured by TCQ and prove the satisfactory reliability of this self-report measure within a Polish population.

Keywords: thought control strategies, suppression, intrusions

\section{Introduction}

The issue of intrusion, which refers to the psychological phenomenon of experiencing undesirable contents such as unwanted thoughts or emotions, is a lively topic in psychological research. In either general or clinical populations, several studies have confirmed that intrusions are psychopathological symptoms of anxiety, obsessive-compulsive disorders, ${ }^{1,2}$ phobias, ${ }^{3}$ post-traumatic stress disorder, ${ }^{4}$ generalized
Szczepanowski

Department of Public Health, Faculty of Health Sciences, Wroclaw Medical University, Wroclaw ul. Kazimierza Bartla 5,

Wroclaw, 5I-6I8, Poland

$\mathrm{Tel}+48717841815$

Fax +48713479359

Email remigiusz.szczepanowski@umed. wroc.pl 
anxiety disorder or depression. ${ }^{5}$ There are still controversies concerning the effectiveness of the psychological mechanisms that deal with intrusions. For instance, Wegner ${ }^{6,7}$ conducted a series of well-known experiments in which participants were instructed to avoid thinking about an imaginary white bear. It was shown that participants' attempts to prevent intrusions led to the paradoxical intensification of intrusions and, as a result, this may exacerbate the frequency of intrusions. On the other hand, Anderson et $\mathrm{al}^{8,9}$ experimentally showed that individual strategies for dealing with intrusions resulted in a reduction in the frequency of experienced intrusions. Other research by Anderson ${ }^{8,9}$ showed that people handled intrusions more effectively when they spent more time spent learning the mechanisms for coping with them. This implies that the problem of understanding intrusions is ongoing and further empirical studies investigating this issue are needed.

To address this research problem, Wells and Davies ${ }^{10}$ proposed a psychometric scale, the Thought Control Questionnaire (TCQ), to measure psychological strategies for coping with intrusions instead of experimentally measuring their effectiveness. This approach focuses on studying individual differences in psychological ways of eliminating intrusions. In fact, studies on strategies for dealing with intrusions are rarely explored in the literature. As Wells and Davies ${ }^{10}$ point out, little is known about how many strategies there are for coping with intrusions. As a result of this research question, they proposed several factors that underlie the psychological strategies measured by the Thought Control Questionnaire (TCQ; Wells \& Davies ${ }^{10}$ ). The idea of using several different coping strategies to construct the TCQ subscales was based on structured interviews from either non-clinical or clinical samples. ${ }^{10}$ In particular, clinical samples originated from individuals suffering from obsessive-compulsive disorder, hypochondria, or generalized anxiety disorder. Ultimately, the interview data were grouped into five factors that indicate distinct psychological strategies for dealing with intrusions: distraction, social control, worry, re-appraisal and punishment. The interrelationships among these factors were weak, which suggests plausible psychological dimensions behind these strategies.

Now, we focus on precise definitions of each strategy. The strategy based on distraction assumes that individuals deal with intrusions by shifting attention to other objects (eg working or pleasant phenomena) instead of focusing on intrusions. ${ }^{10}$ In turn, individuals who use social control tend to talk with other people about intrusions and coping strategies; on the other hand, they may hide intrusions in the course of social interactions. ${ }^{5,10}$ The worrying strategy is based on replacing the content of the intrusion with other unpleasant new thoughts that often are less important and less stressful. Overall, this strategy replaces some worries (intrusions) with less unpleasant ones. As Wells and Davies ${ }^{10}$ stressed, there is an obvious controversy regarding this strategy. Namely, worrying is not always an effective strategy because it may enhance unpleasant phenomena and subsequently increase intrusions. Another strategy for dealing with intrusions is re-appraisal. There are different ways of implementing this strategy: reinterpreting the intrusion content, rational analysis of the content, depreciation of intrusions, or thinking about the reasons for the intrusion. According to Wells and Davies, ${ }^{10}$ it is debatable whether the cognitive appraisal is an effective strategy per se for dealing with intrusions or reducing the stress caused by them. The last strategy distinguished by TCQ is punishment; this strategy is used by individuals to punish themselves for having intrusions, either physically (eg, pinching or slapping) or psychologically (eg getting angry/shouting at oneself for having intrusions or thinking that intrusions will cause something bad to happen).

As mentioned above, the development of TCQ has contributed to a new approach that focuses on several thought control strategies for preventing intrusions, instead of studying a single, general psychological mechanism that shapes the effectiveness of suppressing intrusions. Moreover, it is worth mentioning that TCQ is a relatively complex measure of suppression strategies, as opposed to the other tool measuring phenomena related to suppression, ie White Bear Suppression Inventory (WBSI) measure. ${ }^{7}$ WBSI indicates a two-factor structure in terms of thought control strategy: intrusions (ie frequency of intrusive thoughts) and suppression tendency. ${ }^{11,12}$ This suggests that TCQ seems to be a more reliable measure if one links suppression of intrusions with specific psychological strategies oriented to achieve goal-oriented behavior. $^{13}$

Investigations of individual differences in terms of thought suppression strategies are important from a clinical perspective. These control strategies are especially relevant to improving the treatment of patients who struggle with unwanted intrusions such as obsessions or phobias. ${ }^{13}$ Therefore, TCQ measurements of coping strategies appear to be particularly useful in cross-sectional 
studies that analyze psychopathology. Several studies have found links between thought control strategies assessed by TCQ and psychometric measures of psychopathology. ${ }^{5}$ For instance, it was shown that people with a high predisposition to depression used strategies based on distraction significantly less often. ${ }^{5}$ Other reports indicated that distraction may be negatively associated with general psychopathology related to symptoms of anxiety, depression, obsessional problems, and sleep disturbances. ${ }^{14}$ On the other hand, the punishment strategy correlated positively with depression scores, ${ }^{5}$ general psychopathological symptoms, ${ }^{14}$ and dysfunctional metacognition. ${ }^{15}$ For instance, the worry-based strategy turned out to be positively correlated with two Meta-cognitions questionnaire (MCQ) measures of metacognition: positive beliefs about worry; beliefs about responsibility and superstition. ${ }^{15}$ Some studies suggest that the worry and punishment strategies are positively correlated with dysfunctional beliefs ${ }^{15}$ and psychopathological symptoms such as anxiety, depression, and intrusions. ${ }^{5,10,16,17}$ In turn, re-appraisal was positively associated with four MCQ scales: positive beliefs about worry; negative beliefs about the uncontrollability of thoughts and their danger; beliefs about superstition and responsibility in relation to one's own thoughts; cognitive self-consciousness. ${ }^{15}$ From this psychometric perspective, re-appraisal also seems to be a strategy that contributes to psychopathology; this is the opposite of what one might expect, and, at this point, it should be emphasized that Wells and Davies ${ }^{10}$ propose that re-appraisal can be beneficial if only it is flexible and periodic. It is also important to note that the strategy based on social control was not linked to any of the aforementioned measures of psychopathology. This may be explained by the fact that social control requires either overt conversations with others about intrusions or concealing them during conversations with others. The impact of exhibiting vs concealing intrusions might result in either enhancing or decreasing symptoms, respectively.

Taken together, the previously presented reports have clearly shown that the TCQ is a reliable instrument for investigating thought control strategies in both general (Luciano et al, $2006^{13}$; Wells \& Davies ${ }^{10}$ ) and clinical populations. $^{5,14,16-19}$ Importantly, studies based on TCQ provide compelling evidence that thought control is a multi-dimensional construct, ${ }^{10,18}$ and while some postulated psychological mechanisms may be helpful, others are ineffective when targeting clinical populations. Thus, the main aim of the first study was to test whether the structure of the Polish version of TCQ is consistent with the construct of the original version of this tool. ${ }^{10}$ Given the abovementioned psychometric findings on thought control strategies, we hypothesized that social control and distraction would be negatively associated with dysfunctional metacognition and with symptoms of depression and anxiety. We also expected that worry and punishment would be positively related to psychological distress (depression and anxiety) and dysfunctional metacognitive beliefs. In particular, one might expect that "bad suppressors" would attain high scores on the punishment or worry subscales and would be more vulnerable to the paradoxical effects of suppression (the increased frequency of intrusions) than "good suppressors", who score highly on the distraction subscale.

\section{Method and Materials \\ Study I \\ Participants}

One hundred and seventy-eight individuals (103 females) aged 18 to $57(\mathrm{M}=24.40, \mathrm{SD}=7.80)$ participated in this study. Participants were undergraduate students from the University of Social Sciences and Humanities, Faculty in Wroclaw, University of Lower Silesia, and Wroclaw University of Science and Technology. All participants were informed about the purpose of the study and were asked if they agreed to participate in it (if so, they signed the consent form). Subjects were informed that they could withdraw from the study at any moment without any consequences. Participants with a history of psychiatric or neurological disorders were excluded from this study. The study was accepted by the local ethics committees of the University of Social Sciences and Humanities, Faculty in Wroclaw and the University of Lower Silesia Commission for Ethics of Scientific Research.

\section{Procedure of the Study}

We used the Polish version of the Thought Control Questionnaire (TCQ), which assesses psychological strategies for the control of unpleasant and unwanted thoughts. $^{10}$ The original tool consists of 30 statements that measure five key strategies for controlling unwanted thoughts: Distraction, Social Control, Worry, Punishment, and Re-appraisal. Each item was assessed by participants on a 4-point Likert-type scale: 1 - never, 2 - sometimes, 3 - often and 4 - almost always. 
We adapted the Polish version of the TCQ based on the back-translation procedure. First, a team of two translators with psychological backgrounds (one of them had previously lived in English-speaking countries) who were fluent in English and native in Polish translated the original version into a single Polish version of the tool. Then, three psychologists with expertise in cognitive psychology, and a specialist in the English language (the anglicist) who is familiar with British and American culture evaluated all aspects of the translation and reached an agreement in terms of the items' meanings. Then, participants from the general population were requested to complete the Polish version and evaluate whether it was clear and understandable. Then, two bilingual translators did two back-translations. Next, the team involved in preparing the Polish version of the instrument (two psychologists and the English-language specialist) evaluated its compatibility with the original. The content of the back-translations did not depart from the original version. The instructions and graphical layout of the Polish adaptation are very similar to the original version. The Polish version of TCQ is available upon this downloading link:

https://1 drv.ms/b/s! Atb 1 apRyNNPQg55WB6VD yRsAaSS8Ug?e=FT5FA6.

\section{Statistical Analysis}

To test the structural model, we performed Confirmatory Factor Analyses using the generalized least-squares method with five orthogonal factors found by exploratory factor analyses in previous studies. ${ }^{10}$ The analysis was performed using the bootstrapping technique applied in AMOS..$^{20}$ In this model, each control strategy (the subscale) represented a latent variable, and each item was an indicator variable. The results indicated that the root-mean -square error of approximation (RMSEA) was 0.05 , and the ratio of the chi-square $(\chi 2=609.117, p<0.001)$ to the degrees of freedom $(d f=401)$ was 1.52. These values confirmed the 5 -factor structure of the instrument. Additionally, the GFI value was 0.77 , and adjusted GFI (AGFI) was 0.73; the obtained results indicated a marginal fit of the model (assuming 0.9 as a satisfactory fit) to the data. Moreover, the CFI value was 0.45 , which indicates a poor fit of the model.

Thus, the suitability of the original structure was not supported by our data. Due to unsatisfactory results, an exploratory factor analysis was carried out using data from the second sample.

\section{Study 2 \\ Participants}

The main aim of the second study was to explore the factorial structure of the Polish version of TCQ. One hundred and seventy-six individuals (108 females) aged 18 to $47(\mathrm{M}=23.77, \mathrm{SD}=6.42)$ participated in this study; these were students from the University of Social Sciences and Humanities, Faculty in Wroclaw, University of Lower Silesia, and Wroclaw University of Science and Technology. Each participant signed the informed consent form and was informed that they could withdraw from the study at any moment without any consequences. Participants with a history of psychiatric or neurological disorders were excluded from this study. The study was accepted by the University of Lower Silesia Commission for Ethics of Scientific Research.

\section{Procedure of the Study}

The procedure of the second study was congruent with the procedure of the first study, which is described above.

\section{Concurrent and Divergent Validity of TCQ}

In order to determine the validity of the Polish version of the TCQ, several additional psychological measures were administered to the subjects who took part in Studies 1 and 2 agreed to further continue participating in the validation study. One hundred and forty-seven participants (119 females, $\mathrm{M}=26.79, \mathrm{SD}=8.32$ ) completed the Polish version of the BDI, ${ }^{21}$ The State-Trait Anxiety Inventory $(\mathrm{STAI}-\mathrm{T})^{22}$ and The Meta-Cognitions Questionnaire. ${ }^{23}$

\section{Beck Depression Inventory ${ }^{24}$}

The Polish version of the BDI, ${ }^{21}$ which consists of 21 selfreport items and is commonly used to assess the severity of depressive symptoms. Each item consists of a list of four statements that participants rate from 0 to 3 . The participants are required to choose the sentence that most closely matches the severity of their depressive symptoms over the last 2 weeks. Higher scores represent more severe depressive symptoms. In this study, the reliability of the BDI was high (Cronbach's $\alpha=0.89$ ).

\section{The State-Trait Anxiety Inventory (STAI-T) ${ }^{22}$}

We employed the Polish adaptation of this tool. ${ }^{25}$ This 20item self-report subscale was used to access anxiety level as a personality trait. The participants rated each of the statements on a 4-point scale from 1 ("almost never") to 4 ("almost always"). Higher scores indicate a higher level of 
anxiety. The reliability of this subscale was satisfactory (Cronbach's $\alpha=0.77$ ).

\section{The Meta-Cognitions Questionnaire ${ }^{23}$}

We used the Polish version of the MCQ by Gawęda. ${ }^{26,27}$ The MCQ consists of 65 items which are rated on a 4-point scale from 1 ("Do not agree") to 4 ("Very much agree"). This tool assesses metacognitive beliefs (beliefs about thinking) and includes five different factors: ${ }^{23} 1$ ) MCQ1 - positive beliefs about worrying (eg "Worrying helps me to plan the future more effectively"), which describes the belief that perseverative thinking is useful (Cronbach's $\alpha=0.94 ; 2$ ) MCQ2 - negative beliefs about the uncontrollability of thoughts and danger (eg "I cannot ignore my worrying thoughts") and indicates beliefs that perseverative thinking is uncontrollable and dangerous (Cronbach's $\alpha=0.94$ ); 3); MCQ3 indicates beliefs about cognitive confidence, ie confidence in one's own cognitive processes in terms of attention and memory (eg "I have little confidence in my memory for words and names") (Cronbach's $\alpha=0.87)$; 4) MCQ4 indicates general negative beliefs (including superstition, punishment and responsibility) which imply that thoughts must not be uncontrolled (eg "I will be punished for not controlling certain thoughts") (Cronbach's $\alpha=0.85$ ); and 5) MCQ5 cognitive self-consciousness (eg "I am constantly aware of my thinking"), which is found in individuals who tend to monitor their own thoughts and focus attention on internal experiences (Cronbach's $\alpha=.76$ ).

\section{Statistical Analysis}

In order to identify the factor structure of the Polish version of the TCQ inventory, a Principal Component Analysis (PCA) was performed. To determine the factorability of the correlation matrix, we used Bartlett's test of sphericity and Kaiser-Meyer-Olkin's (KMO) measure of sampling adequacy. Bartlett's test yielded a chi-square value of 2038.85 (435), $p<0.001$, indicating that the data were suitable for the factor analysis. The KMO was 0.746 , which indicated that factor analysis was a suitable method according to the conventional assumption that a value above 0.50 ensures factorability. A principal component analysis with oblique (Direct Oblimin) rotation and Kaiser Normalization was performed with the assumption that there were significant correlations between factors. ${ }^{10}$ To distinguish the underlying factors of the inventory, we took into account the Kaiser criterion and considered all factors with eigenvalues greater than one. We also performed a visual inspection of the break in the slope on the scree $\operatorname{plot}^{28}$ and interpreted the resulting factorial solution. Finally, the reliability of the Polish version of TCQ was assessed by calculating the Cronbach's alpha reliability coefficients for each factor.

\section{Results Exploratory Factor Analysis}

The Kaiser criterion suggested that there were seven factors with eigenvalues greater than 1.0. The first seven observed eigenvalues were 4.66, 3.86, 3.31, 2.20, 1.46, 1.32 and 1.26. We found that seven factors accounted for $60.25 \%$ of the total variance. However, this method of selecting factors may be inaccurate $^{29}$ and might result in too many factors being retained. ${ }^{30}$ Further inspection of the break in the slope on the scree plot from Study 2 indicated that five factors should be considered in the final solution (see Figure 1). The five-factor structure was mostly in line with the original structure of the $\mathrm{TCQ}^{10}$ and has been recognized as being psychologically interpretable. Therefore, the five-factor solution of the Polish version was finally accepted.

Exploratory principal components analysis was performed on the 30-item TCQ. We employed PCA combined with direct oblimin rotation and Kaiser normalization to identify the five-factor solution of the scale. It was observed that five factors explained $51.64 \%$ of the total variance. The factor loadings for the five-factor solution are presented in Table 1. We included statements with factorial loadings above the value of $0.4 .{ }^{31}$ Note that some items also met the criteria for inclusion in other factors. Finally, these items were included in the scale with higher factorial loadings, apart from item 3, which loaded either factor $1(0.402)$ or 2 $(-.523)$. Finally, this particular item was included in factor 1 to preserve consistency with other statements that structured this factor. All items met the criteria for inclusion in each selected factor, and no single statement was removed from the inventory. The final structure of the instrument was composed of the following subscales: the first subscale included seven items, the second had six items, the third included six items (three items are scored in a reverse manner: $5,8,12$ ), the fourth measure included 6 items, and the fifth consisted of 5 items. The total variance of the fivefactor solution was $51.86 \%$, collapsed into particular components equal to $16.09 \%$ for the first factor, $13.42 \%$ for the second factor, $10.97 \%$ for the third factor, $6.68 \%$ for the fourth factor, and $4.69 \%$ for the fifth factor. 


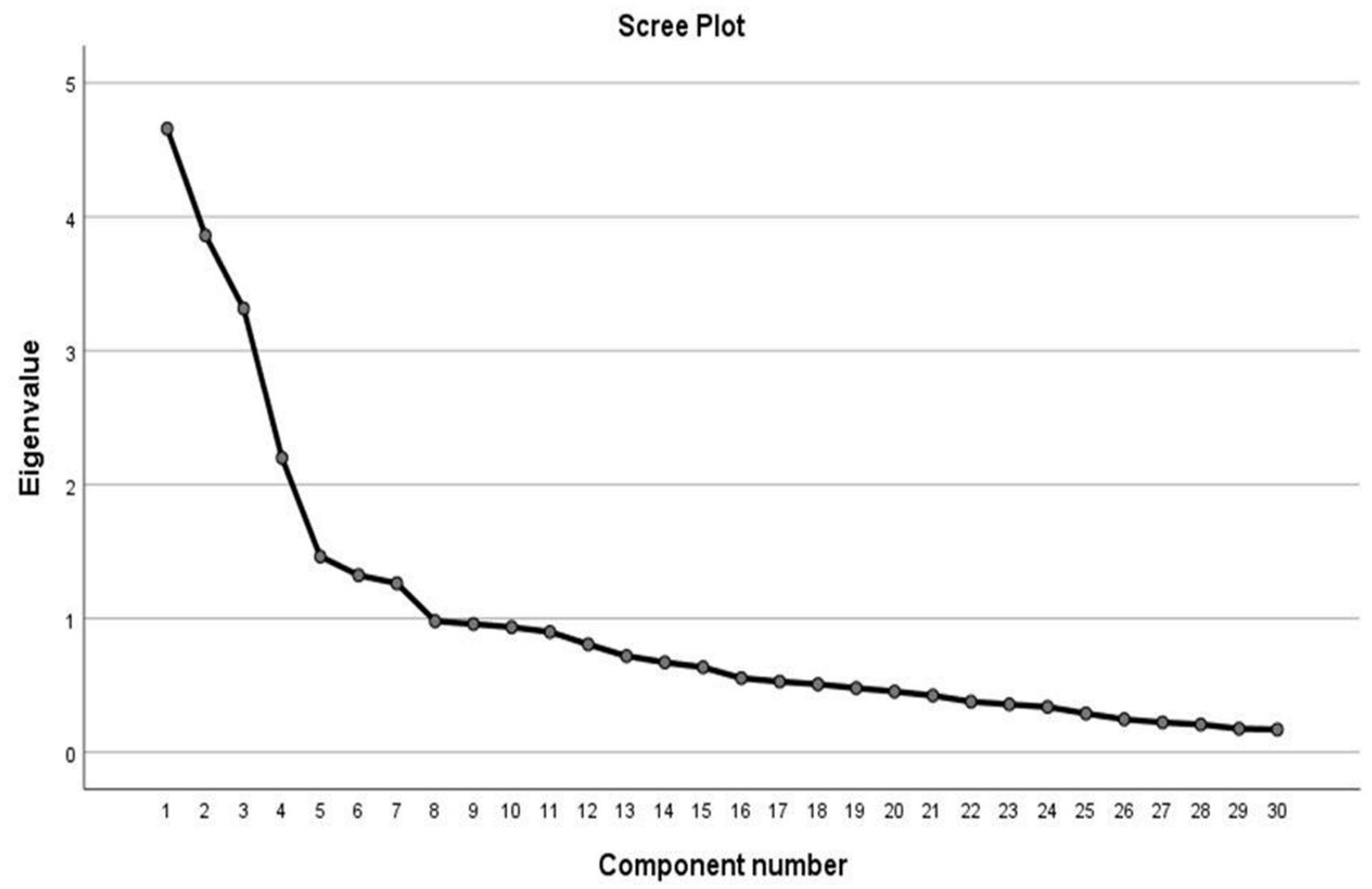

Figure I Scree plot of eigenvalues after principal component analysis. The number of components is on the $x$-axis and eigenvalue on the $y$-axis.

The scale labels were termed as follows: 1) Worry (items 4, 7, 18, 22, 24 and 26); 2) Distraction (items 1, 3(r), 9, 16, 19, 21 and 30); 3) Social Control (items 5(r), 8(r), 12(r), 17, 25 and 29); 4) Re-appraisal (items 10, 14, 20, 23 and 27); 5) Punishment (items 2, 6, 11, 13, 15 and 28). Items 3, 5, 8 and 12 were scored in a reverse manner. In fact, the content of the five-factor rotated solution turned out to be very similar to the original version of TCQ. ${ }^{10}$ There was only a difference for item 3, which was originally included in the Re-appraisal subscale in the original TCQ, while in the Polish adaptation of the TCQ measure this item was reversed and moved to the Distraction subscale.

\section{Internal Consistency of Subscales}

In the next step, we assessed the reliability of the TCQ subscales by calculating the Cronbach's alpha reliability coefficients for each factor. The following values of the Cronbach's alphas were obtained: 1) Punishment yielded $\alpha$ $=0.725 ; 2$ ) Distraction yielded $\alpha=0.688$ (if item 3 were removed for the scale, $\alpha=0.704)$; 3) Social Control yielded $\alpha=0.780$; 4) Worry yielded $\alpha=0.788$; 5) Reappraisal yielded $\alpha=0.70$. The reliability of all five scales was satisfactory, thus indicating the homogeneous structure of the particular scales. In the case of Distraction, reliability reached a value of 0.688 , but this result was considered satisfactory (slightly below 0.7 ).

\section{Subscale Inter-Correlations}

The intercorrelations among the individual factors and descriptive statistics $(\mathrm{N}=176)$ are presented in Table 2. It was found that Distraction was positively related to Reappraisal and negatively to Punishment. Punishment was also positively associated with Worry and Re-appraisal. The correlation coefficients were relatively low. These results confirmed to some extent the assumption that the TCQ subscales measure control strategies that are distinct psychological constructs. It is also worth commenting on our inter-correlation results, comparing them to previous analyses obtained by Wells and Davies ${ }^{10}$. As in the original version of TCQ, our results provide empirical evidence that the subscales represent separate psychological dimensions in terms of suppression strategies. Here, we provide a summary of comparing our results with those of Wells and Davies, ${ }^{10}$ (see Table 2). It was shown that only two pairs of 
Table I The Results of Principal Component Analysis and Factor Loadings of the 30-Item Thought Control Questionnaire (N = I76) for the Five-Factor Solution

\begin{tabular}{|c|c|c|c|c|c|}
\hline Item & Factor I Worry & Factor 2 Distraction & Factor 3 Social Control & Factor 4 Re-Appraisal & Factor 5 Punishment \\
\hline 26 & $0.80 \mathrm{I}$ & & & & \\
\hline 18 & 0.743 & & & & \\
\hline 4 & 0.686 & & & & \\
\hline 7 & 0.659 & & & & \\
\hline 24 & 0.632 & & & & \\
\hline 22 & 0.570 & 0.500 & & & \\
\hline 21 & & 0.782 & & & \\
\hline 16 & & 0.697 & & & \\
\hline 1 & & 0.636 & & & \\
\hline 19 & & 0.595 & & & \\
\hline 3 & & -0.557 & & & \\
\hline 9 & & 0.446 & & & \\
\hline 30 & & 0.426 & & & \\
\hline 29 & & & 0.838 & & \\
\hline 17 & & & 0.825 & & \\
\hline 25 & & & $0.8 \mathrm{I} I$ & & \\
\hline 8 & & & -0.691 & & \\
\hline 12 & & & -0.664 & & \\
\hline 5 & & & -0.600 & & \\
\hline 27 & & & & $0.67 I$ & \\
\hline 23 & & & & 0.644 & \\
\hline 14 & & & & 0.628 & \\
\hline 10 & & & & 0.604 & \\
\hline 20 & & & & 0.435 & \\
\hline 13 & & & & & 0.726 \\
\hline 15 & & & & & 0.668 \\
\hline 6 & & & & & 0.586 \\
\hline II & & & & & 0.583 \\
\hline 2 & & & & & 0.578 \\
\hline 28 & & & & & 0.357 \\
\hline
\end{tabular}

Notes: The items included in particular sub-scales are presented in bold. Values above 0.4 were established as inclusion criteria. 
Table 2 Pearson Product-Moment Correlations Between Individual Subscales and Descriptive Statistics for the TCQ Factors ( $\mathrm{N}=$ 176)

\begin{tabular}{|l|l|l|l|l|l|l|}
\hline & $\mathbf{2}$ & $\mathbf{3}$ & $\mathbf{4}$ & $\mathbf{5}$ & $\mathbf{M}$ & SD \\
\hline (1) Worry & -0.028 & -0.035 & 0.089 & $0.370^{* * *}$ & 9.81 & \\
(2) Distraction & & 0.076 & $0.365 * * *$ & $-0.198^{* *}$ & 17.81 & 3.02 \\
(3) Social Control & & & 0.037 & -0.074 & 14.51 & 3.68 \\
(4) Reappraisal & & & $0.163^{*}$ & 12.44 & 2.15 \\
(5) Punishment & & & & & 10.58 & 3.42 \\
\hline
\end{tabular}

Notes: $*_{p}<0.05 ; * *_{p}<0.01 ; * * *_{p}<0.001$.

intercorrelation differed significantly. The congruent values of intercorrelations were observed for the pairs of the following dimensions (all remaining at $p>0.05$ ): Worry and Distraction $\left(0.09^{\mathrm{a}}\right.$ (a: the original TCQ value) vs $-.03^{\mathrm{b}}$ (b: the present version of TCQ), Worry and Social Control $\left(-.01^{\mathrm{a}}\right.$ vs $\left.-.04^{\mathrm{b}}\right)$, Worry and Re-appraisal $\left(-.02^{\mathrm{a}}\right.$ vs $\left.0.09^{\mathrm{b}}\right)$, Worry and Punishment $\left(0.27^{\mathrm{a}}\right.$ vs $\left.0.37^{\mathrm{b}}\right)$, Social Control and Distraction $\left(-.02^{\mathrm{a}}\right.$ vs $\left.0.08^{\mathrm{b}}\right)$, Social Control and Re-appraisal $\left(0.13^{\mathrm{a}}\right.$ vs $\left.0.04^{\mathrm{b}}\right)$, Social Control and Punishment $\left(0.04^{\mathrm{a}}\right.$ vs $\left.-.07^{\mathrm{b}}\right)$, Punishment and Re-appraisal $\left(0.13^{\mathrm{a}}\right.$ vs $\left.0.16^{\mathrm{b}}\right)$. Discrepancies between the values of the intercorrelations between the original and present versions of TCQ were observed for the following pairs of the dimensions: Distraction and Re-Appraisal $\left(0.10^{\mathrm{a}}\right.$ vs $\left.0.37^{\mathrm{b}} ; p<0.01\right)$, Distraction and Punishment $\left(0.16^{\mathrm{a}}\right.$ vs $\left.-.20^{\mathrm{b}} ; p<0.001\right)$.

\section{Concurrent and Divergent Validity of TCQ}

The final analysis aimed to determine the validity of the Polish version of the TCQ based on its correlations with psychological measures of BDI, ${ }^{24}$ STAI-T $^{22}$ and MCQ. ${ }^{26,27}$ Table 3 contains the Pearson product-moment correlations that were computed between the TCQ subscales and the BDI, STAI-T and MCQ factors.
The analysis revealed a significant negative correlation between the strategy based on social control and the MCQ4 factor $(r=-.155 ; p<0.05)$. There was also a significant negative correlation between distraction and symptoms of depression $(r=-.346, p<0.001)$, anxiety $(r=-.157, p<0.05)$, and dysfunctional metacognition linked with positive beliefs about worrying (MCQ1) $(r=$ $-.150, p<0.05)$, negative beliefs about the uncontrollability of thoughts and danger (MCQ2) $(r=-.348, p<0.001)$, beliefs about cognitive confidence (MCQ3) $(r=-.255, p<$ $0.001)$ and general negative beliefs $(r=-.199, p<0.01)$. These results suggest that deficits in the strategy based on distraction were linked with depression, anxiety and dysfunctional metacognition. We also observed that the strategy based on worrying positively correlated with depression, $r=0.137, p=0.096$ ) and all dysfunctional metacognitive beliefs measured with MCQ, ie MCQ1, $r=$ 0.263, $p<0.001$; MCQ2, $r=0.237, p<0.01$; MCQ3 $r=$ $0.241, p<0.001$; MCQ4, $r=0.333, p<0.001$ and MCQ5, $r=0.182, p<0.01$. The punishment subscale was positively correlated with both depression $(r=0.307, p<$ $0.001)$ and trait anxiety $(r=0.375, p<0.001)$. There were also positive associations between punishment and all the metacognitive factors: MCQ1, $r=0.411, p<0.001$;

Table 3 Pearson Product-Moment Correlations Between the TCQ Subscales and the BDI, STAI-T and MCQ Factors

\begin{tabular}{|l|l|l|l|l|l|l|l|}
\hline & BDI & Anxiety Trait & \multicolumn{4}{l|}{ Metacognitive Beliefs (n = 208) } \\
\hline TCQ (n= 208) & $\mathbf{( n = 1 4 9 )}$ & $\mathbf{( n = 2 0 4 )}$ & MCQI & MCQ2 & MCQ3 & MCQ4 \\
\hline Social Control & -0.121 & -0.094 & -0.061 & -0.053 & -0.038 & $-0.155^{*}$ & -0.018 \\
Distraction & $-0.346^{* * *}$ & $-0.157^{*}$ & $-0.150^{*}$ & $-0.348^{* * *}$ & $-0.255^{* * *}$ & $-0.199^{* *}$ \\
Worry & $0.137^{\wedge}$ & 0.069 & $0.263^{* * *}$ & $0.237^{* *}$ & $0.241^{* * *}$ & $0.333^{* * *}$ & -0.007 \\
Punishment & $0.307^{* * *}$ & $0.375^{* * *}$ & $0.411^{* * *}$ & $0.606^{* * *}$ & $0.303^{* * *}$ & $0.540^{* * *}$ \\
Reappraisal & -0.110 & $0.185^{* *}$ & 0.102 & $0.158^{*}$ & $0.178^{* *}$ & $0.328^{* * *}$ \\
Total TCQ & -0.112 & 0.112 & $0.185^{* *}$ & $0.183^{* *}$ & $0.124^{\wedge}$ & $0.225^{* *}$ & $0.378^{* * *}$ \\
\hline
\end{tabular}

Notes: ${ }^{*} p<0.05 ; * * p<0.01 ;{ }^{* * *} p<0.00 \mathrm{I} ; \wedge 0.10>p>0.05$ (a marginal trend toward significance).

Abbreviations: BDI, Beck Depression Inventory scores; TCQ, Thought Control Questionnaire; MCQI, positive beliefs about worrying; MCQ2, negative beliefs about the uncontrollability of thoughts and danger; MCQ3, beliefs about cognitive confidence; MCQ4, general negative beliefs (including superstition, punishment and responsibility); MCQ5, cognitive self-consciousness. 
MCQ2, $r=0.606, p<0.001 ;$ MCQ3, $r=0.303, p<0.001$; MCQ4, $r=0.540, p<0.001$; MCQ5, $r=0.328, p<0.001$. We also found a positive association between reappraisal and trait anxiety $(r=0.185, p<0.01)$ and dysfunctional metacognition linked with MCQ2: $r=0.158, p<0.05$; MCQ3 $-r=0.178, p<0.01$; MCQ4 $-r=0.207, p<0.01$; MCQ5 $-r=0.378, p<0.001$.

\section{Discussion}

The aim of this article is to analyze the psychometric properties of the Polish version of the TCQ scale by investigating its factor structure and validating TCQ outcome measures with other self-report measures associated psychologically or clinically with suppression in the general population. Our results suggest either the five- or seven-factor solutions for the inventory. Parallel analysis and the theoretical interpretability of the Polish version showed that the optimal solution was the five-factor structure that explains $51.86 \%$ of the variance. Given the similarities between the factor structures of the present Polish and the original TCQ versions, we labeled the subscales the same way as in the primary version of TCQ: 1) punishment ( $\alpha=0.74) ; 2)$ distraction $(\alpha=0.76) ; 3)$ social control $(\alpha=0.86)$; 4) worry $(\alpha=0.78) ; 5)$ re-appraisal $(\alpha=0.70)$. Note that the obtained reliabilities of the subscales are comparable to the Cronbach's alphas from the original TCQ: Distraction $=0.72$; Social Control $=0.79$; Worry $=$ 0.71; Punishment $=0.64$; Re-appraisal $=0.67 .{ }^{9}$ This indicates that all subscales of the Polish version are characterized by satisfactory values of Cronbach's alpha and constitute a reliable measure of individual strategies to control unpleasant and unwanted thoughts.

In addition to analysis of the factor structure that conforms to the Polish version of TCQ, we examined how the TCQ measures were associated with other psychometric scales in order to validate the Polish TCQ version and to study intrusions and psychopathological symptoms in the general population. The vast majority of our results were consistent with the findings published so far. ${ }^{14,15}$ For example, it was shown that anxiety and depression were negatively associated with distraction among patients with PTSD. ${ }^{5}$ Our results confirmed the previous findings ${ }^{5,14,16,32}$ and suggest that the strategy based on distraction may be linked with psychopathology; it was negatively correlated with depression and trait anxiety and low levels of dysfunctional metacognition (ie beliefs including positive beliefs - about worrying, negative beliefs about the uncontrollability of thoughts and danger, beliefs about cognitive confidence, and general negative beliefs). Thus, the distraction strategy may effectively suppress unwanted thoughts. For example, Abramowitz et $\mathrm{al}^{16}$ suggest that distraction enhances the accuracy and benefits of assessments of intrusions. Unwanted thoughts are experienced as less harmful; therefore, distractions (such as keeping busy) may inhibit excessive threatrelated appraisals. ${ }^{16}$

Our findings also indicate that punishment tends to be used by more depressed and anxious individuals and people with higher levels of dysfunctional metacognitive beliefs (all MCQ scales). These results are consistent with previous studies that indicated that the tendency to use punishment to suppress unwanted thoughts is related to the severity of symptoms of $\mathrm{OCD},{ }^{16,32}$ depression, anxiety and intrusions. ${ }^{5,10}$

As was expected, worrying turned out to be positively associated with all dysfunctional metacognitive beliefs. Indeed, previous studies have indicated that worry is associated with anxiety disorders, ${ }^{33}$ increased depression, obsessive thoughts and pathological worry. ${ }^{16,17}$ Worry is also an important predictor of post-traumatic stress disorder (PTSD). ${ }^{34}$ It is suggested that the excessive use of worrying to suppress unwanted thoughts favors retaining or even increasing stress, ${ }^{16}$ thus resulting in an intensification of intrusions. ${ }^{35}$

Regarding the re-appraisal strategy, it turned out to be associated with negative beliefs about the uncontrollability of thoughts and their danger, beliefs about cognitive confidence, general negative beliefs, and cognitive selfconsciousness. The frequent use of this strategy of interpreting situations and events significantly increased trait anxiety in participants. These results suggest that reappraisal is an ineffective thought-control strategy. Considering the findings of previous studies, there is some ambiguity related to the effectiveness of reappraisal. Some researchers suggest that re-appraisal leads to a reduction of negative emotions ${ }^{35}$ and consider it to be an adaptive coping strategy. For example, studies in depressed and PTSD populations have shown that recovering patients were more likely to use re-appraisal. ${ }^{5}$ This control strategy is also associated with reducing the severity of depression and intrusions among people with PTSD and depression. ${ }^{5}$ On the other hand, some studies suggest that patients with anxiety disorders are more likely to use re-appraisal and punishment than other strategies. ${ }^{16}$ The use of re-appraisal is associated with higher levels of anxiety ${ }^{16}$ increased obsessive-compulsive symptoms, and 
lack of control over thoughts. ${ }^{36}$ At the same time, the same authors ultimately include reassessment in the group of adaptive strategies. ${ }^{36}$ Thus, research on the effectiveness of this strategy is ambiguous. Some researchers suggest that the use of re-appraisal requires the involvement of more cognitive resources to transform an emotional state into another. ${ }^{5,37}$ When individuals have enough cognitive resources, this strategy can probably lead to effective suppression of unwanted thoughts. However, in cognitive load conditions, re-appraisal may be ineffective due to a lack of resources to change the current emotional state. ${ }^{5,37}$

The last suppression strategy measured by TCQ was social control. Contrary to the previous works, ${ }^{16,17}$ we found no association between this strategy and depression and anxiety. The relationship between social control and psychopathology is unclear. For example, Coles and Heimberg ${ }^{17}$ found that social control is associated with a reduction in anxiety symptoms and a higher level of life satisfaction. Our results showed that there is a negative correlation between social control and the MCQ measure, ie negative beliefs that usually contribute to the development of psychopathology.

It is worth mentioning that several studies based on exploratory factor analysis (EFA) have also confirmed the five-factor structure of the TCQ for either nonclinical subjects $^{9,38}$ or clinical samples. ${ }^{5}$ A German validation study found that the factorial structure was the same as that presented by the original version of TCQ. ${ }^{39}$ The same structure was confirmed in the Spanish version, although the removal of a few items was needed to preserve the original five-factor structure. ${ }^{13}$ On the other hand, some studies failed to replicate the five-factor structure. For instance, $\operatorname{Re}^{18}$ revealed the existence of a four-factor structure of TCQ in which the re-appraisal and distraction subscales loaded one factor.

One may note that our findings are in line with previous works ${ }^{14,15}$ which showed that distraction seems to be an effective coping strategy, while re-appraisal represents a non-adaptive form of coping. A further interpretation of this finding might reflect the psychoanalytic approach, which suggests a variety of defense mechanisms against intrusions that are mainly aimed at preserving our psychological well-being. ${ }^{40,41}$ Along with previous results, ${ }^{14,15}$ our study seems to support the claim that the thought control strategy has a defensive nature. In this fashion, distraction represents a typical defense mechanism in that it is usually aimed at muting unpleasant thoughts or feelings. On the other hand, re-appraisal more resembles coping mechanisms that are intended to realize the causes of the unpleasant experience.

It should be noted that the present study also has some limitations. The research was conducted on university students, but the instrument can be also targeted at clinical populations that are unable to effectively suppress intrusions. Indeed, it has been proven that unwanted thought suppression plays an important role in the etiology and maintenance of a variety of mental disorders. ${ }^{42-45}$ In particular, the most counterproductive strategies that result in intrusions seem to be punishment and worry. Compared with a healthy population, these strategies are mostly used by patients with obsessive-compulsive disorder (OCD) and are also related to OCD severity ${ }^{32}$ and other psychopathological measures. ${ }^{9,46,47}$ Therefore, further investigation should be carried out to investigate the factor structure of TCQ in clinical populations. Further research is also desirable to investigate whether the specific thought control measured by the Polish version of TCQ can be used to predict paradoxical effects of suppression. ${ }^{11}$ Another limitation of our research was the sample population, because of the unbalanced gender and the age of the undergraduate students. Therefore, to make more general conclusions further investigations of TCQ are needed adjusting the sample population for both factors.

To sum up, our study provides evidence that supports the five-factor structure of TCQ and proves the satisfactory reliability of this measure. Our research results confirm that TCQ is useful as a self-report measure of thought-control strategies within a Polish population. The results of the comparison between the Polish version of TCQ and other psychopathological measures are similar to previous works, ${ }^{14,15}$ which supports the validity of the Polish version of TCQ.

\section{Acknowledgments}

This research has been supported by the National Science Center (Poland) and funded under award number 2014/15/ B/HS6/03834 to R.S.

\section{Disclosure}

The authors report no conflicts of interest for this work.

\section{References}

1. Rachman S, de Silva P. Abnormal and normal obsessions. Behav Res Ther. 1978;16(4):233-248. doi:10.1016/0005-7967(78)90022-0 
2. Salkovskis PM. Cognitive-behavioural factors and the persistence of intrusive thoughts in obsessional problems. Behav Res Ther. 1989;27 (6):677-682. doi:10.1016/0005-7967(89)90152-6

3. Kingsep P, Page A. Attempted suppression of social threat thoughts: differential effects for social phobia and healthy controls? Behav Res Ther. 2010;48(7):653-660. doi:10.1016/j.brat.2010.03.018

4. Horowitz MJ. Stress Response Syndromes. NY: Jason Aronson; 1976.

5. Reynolds M, Wells A. The thought control questionnaire-psychometric properties in a clinical sample, and relationships with PTSD and depression. Psychol Med. 1999;29(5):1089-1099. doi:10.1017/ s003329179900104x

6. Wegner DM. Ironic processes of mental control. Psychol Rev. 1994;101(1):34-52. doi:10.1037/0033-295x.101.1.34

7. Wegner DM, Schneider DJ, Carter SR, White TL. Paradoxical effects of thought suppression. J Pers Soc Psychol. 1987;53(1):5-13. doi:10.1037//0022-3514.53.1.5

8. Anderson MC, Green C. Suppressing unwanted memories by executive control. Nature. 2001;410(6826):366-369. doi:10.1038/ 35066572

9. Anderson MC, Levy BJ. Suppressing unwanted memories. Curr Dir Psychol Sci. 2009;18(4):189-194. doi:10.1111/j.14678721.2009.01634.x

10. Wells A, Davies MI. The thought control questionnaire: a measure of individual differences in the control of unwanted thoughts. Behav Res Ther. 1994;32(8):871-878. doi:10.1016/0005-7967(94)90168-6

11. Cichoń E, Szczepanowski R, Niemiec T. Polish version of White Bear Suppression Inventory (WBSI) by Wegner and Zanakos: factor analysis and reliability. Psychiatr Pol. 2020;54(1):125-135. doi: $10.12740 / \mathrm{pp} / 93493$

12. Höping W, de Jong-meyer R. Differentiating unwanted intrusive thoughts from thought suppression: what does the white bear suppression inventory measure? Pers Individ Dif. 2003;34 (6):1049-1055. doi:10.1016/s0191-8869(02)00089-2

13. Luciano JV, Belloch A, Algarabel S, Tomás JM, Morillo C, Lucero M. Confirmatory factor analysis of the white bear suppression inventory and the thought control questionnaire: a comparison of alternative models. Eur J Psychol Assess. 2006;22(4):250-258. doi:10.1027/1015-5759.22.4.250

14. Rassin E, Diepstraten P. How to suppress obsessive thoughts. Behav Res Ther. 2003;41(1):97-103. doi:10.1016/s0005-7967(02)00043-8

15. García-Montes JM, Cangas A, Pérez-Álvarez M, Fidalgo AM, Gutiérrez $\mathrm{O}$. The role of meta-cognitions and thought control techniques in predisposition to auditory and visual hallucinations. $\mathrm{Br} \mathrm{J} \mathrm{Clin}$ Psychol. 2006;45(3):309-317. doi:10.1348/014466505X66755

16. Abramowitz J, Whiteside S, Kalsy S, Tolin D. Thought control strategies in obsessive-compulsive disorder: a replication and extension. Behav Res Ther. 2003;41(5):529-540. doi:10.1016/ s0005-7967(02)00026-8

17. Coles ME, Heimberg RG. Thought control strategies in generalized anxiety disorder. Cognit Ther Res. 2005;29(1):47-56. doi:10.1007/ s10608-005-1647-x

18. Ree MJ. The thought control questionnaire in an inpatient psychiatric setting: psychometric properties and predictive capacity. Behav Modif. 2010;27(4):212-226. doi:10.1375/bech.27.4.212

19. Rosenthal MZ, Cukrowicz KC, Cheavens JS, Lynch TR. Selfpunishment as a regulation strategy in borderline personality disorder. $J$ Pers Disord. 2006;20(3):232-246. doi:10.1521/ pedi.2006.20.3.232

20. Arbuckle JL. Amos (Version 25.0) [Computer Program]. Chicago: IBM SPSS; 2014.

21. Parnowski T, Jernajczyk W. Beck's depression inventory in the rating of mood in normal individuals and in patients with affective disorders. Psychiatr Pol. 1977;11(4):417-425.

22. Spielberger CD, Gorsuch RL, Lushene PR, Vagg PR, Jacobs GA. Manual for State-Trait Anxiety Inventory for Adults. Menlo Park, CA: Consulting Psychologists Press, Mind Garden; 1983.
23. Cartwright-Hatton S, Wells A. Beliefs about worry and intrusions: the meta-cognitions questionnaire and its correlates. J Anxiety Disord. 1997;11(3):279-296. doi:10.1016/s0887-6185(97)00011-x

24. Beck AT, Ward CH, Mendelson M, Mock J, Erbaugh J. An inventory for measuring depression. Arch Gen Psychiatry. 1961;4(6):561-571. doi:10.1001/archpsyc.1961.01710120031004

25. Sosnowski T, Wrześniewski K, Jaworoska A, Ferenc D. STAI-State and Trait Inventory [In Polish]. Warsaw, Poland: Pracownia Testow Psychologicznych; 2002.

26. Gawęda Ł. Metacognitions Questionnaire [In Polish]. Unpublished Document. Warsaw, Poland: Medical University of Warsaw; 2007.

27. Gawęda $Ł$, Kokoszka A. A relationship between hallucination proneness and character and temperament: a mediating role of meta-cognitive beliefs in a non-clinical sample. Psychiatry Res. 2013;208(2):183-185. doi:10.1016/j.psychres.2013.01.027

28. Cattell RB. The scree test for the number of factors. Multivariate Behav Res. 1966;1(2):245-276. doi:10.1207/s15327906mbr0102_10

29. Velicer WF, Jackson DN. Component analysis versus common factor-analysis - some further observations. Multivariate Behav Res. 1990;25(1):97-114. doi:10.1207/s15327906mbr2501_12

30. Osborne JW, Costello AB, Kellow JT. Best practices in exploratory factor analysis. Best Pract Quant Methods. 2008;86-99. doi:10.4135/ 9781412995627.d8

31. Field AP. Discovering Statistics Using IBM SPSS Statistics. London: Sage; 2013.

32. Amir N, Cashman L, Foa EB. Strategies of thought control in obsessive-compulsive disorder. Behav Res Ther. 1997;35(8):775IN1. doi:10.1016/s0005-7967(97)00030-2

33. Borkovec TD, Ray WJ, Stober J. Worry: a cognitive phenomenon intimately linked to affective, physiological, and interpersonal behavioral processes. Cognit Ther Res. 1998;22(6):561-576. doi:10.1023/ A:1018790003416

34. Holeva V, Tarrier N, Wells A. Prevalence and predictors of acute stress disorder and PTSD following road traffic accidents: thought control strategies and social support. Behav Ther. 2001;32(1):65-83. doi:10.1016/s0005-7894(01)80044-7

35. Fergus TA, Wu KD. Is worry a thought control strategy relevant to obsessive-compulsive disorder? J Anxiety Disord. 2010;24 (2):269-274. doi:10.1016/j.janxdis.2009.12.003

36. Mckay D, Greisberg S. Specificity of measures of thought control. J Psychol. 2002;136(2):149-160. doi:10.1080/00223980209604146

37. Sheppes G, Catran E, Meiran N. Reappraisal (but not distraction) is going to make you sweat: physiological evidence for self-control effort. Int $J$ Psychophysiol. 2009;71(2):91-96. doi:10.1016/j. ijpsycho.2008.06.006

38. Lucero M. Pensamientos Intrusivos en Población General. Intrusive Thoughts in the Normal Population [tesis doctoral]. Spain: Facultad de Psicología, Universidad de Valencia; 2002.

39. Fehm L, Hoyer J. Measuring thought control strategies: the thought control questionnaire and a look beyond. Cognit Ther Res. 2004;28 (1):105-117. doi:10.1023/b:cotr.0000016933.41653.dc

40. Freud S. Collected Papers. Vol. 4. London: Hogarth Press; 1925.

41. Montada L. Justice, equity, and fairness in human relations. In: Millon T, Lerner MJ, Weiner IB, editors. Handbook of Psychology: Personality and Social Psychology. Vol. 5. New Jersey: John Wiley \& Sons, Inc; 2003.

42. Palfai TP, Monti PM, Colby SM, Rohsenow DJ. Effects of suppressing the urge to drink on the accessibility of alcohol outcome expectancies. Behav Res Ther. 1997;35(1):59-65. doi:10.1016/ s0005-7967(96)00079-4

43. Tolin DF, Abramowitz JS, Przeworski A, Foa EB. Thought suppression in obsessive-compulsive disorder. Behav Res Ther. 2002;40 (11):1255-1274. doi:10.1016/s0005-7967(01)00095-x

44. Wenzel A, Barth TC, Holt CS. Thought suppression in spider-fearful and nonfearful individuals. J Gen Psychol. 2003;130(2):191-205. doi:10.1080/00221300309601284 
45. Wenzlaff RM, Wegner DM, Roper DW. Depression and mental control: the resurgence of unwanted negative thoughts. J Pers Soc Psychol. 1988;55(6):882-892. doi:10.1037/0022-3514.55.6.882

46. Guthrie R, Bryant R. Attempting suppression of traumatic memories over extended periods in acute stress disorder. Behav Res Ther. 2000;38(9):899-907. doi:10.1016/s0005-7967(99)00120-5
47. Wells A, Papageorgiou C. Worry and the incubation of intrusive images following stress. Behav Res Ther. 1995;33(5):579-583. doi:10.1016/0005-7967(94)00087-z

\section{Publish your work in this journal}

Psychology Research and Behavior Management is an international, peer-reviewed, open access journal focusing on the science of psychology and its application in behavior management to develop improved outcomes in the clinical, educational, sports and business arenas. Specific topics covered in the journal include: Neuroscience, memory and decision making; Behavior modification and management; Clinical applications; Business and sports performance management; Social and developmental studies; Animal studies. The manuscript management system is completely online and includes a very quick and fair peer-review system, which is all easy to use. Visit http://www. dovepress.com/testimonials.php to read real quotes from published authors. 